\title{
MulTimodel CONTROL AND FUZZY OPTIMIZATION OF AN INDUCTION MOTOR
}

\author{
Mehdi Dhaoui $^{1,3}$, Makhlouf Laakam ${ }^{1,2}$, Lassaad Sbita ${ }^{1,3}$ \\ ${ }^{1}$ Research unit of Photovoltaic, Wind and Geothermal Systems \\ ${ }^{2}$ Higher Institute of Technological Studies of Djerba \\ ${ }^{3}$ National Engineering School of Gabes (ENIG)-Zrig 6029 Gabes, Tunisia. \\ DhaouimeYahoo.fr \\ laakam_makhlouf@yahoo.fr
}

\begin{abstract}
Classical indirect field-oriented control is highly sensitive to uncertainties in the rotor resistance of the induction motor. This sensitivity can be reduced by combining two different methods to compute the stator electrical frequency. Fuzzy logic is used to combine both methods to obtain a compromise which reduces the flux control sensitivity to electrical parameter errors at each operating point. The design of the fuzzy logic block is based on a theoretical sensitivity analysis taking magnetic saturation into account, in simulations. In this paper, the performance of the proposed control algorithm is theoretically studied. The predictions are validated by considering the stator current variations, to develop a given steady-state torque, induced by the imperfect flux control.
\end{abstract}

\section{KEYWORDS}

Fuzzy logic, induction motor drives, parameter uncertainty, robustness.

\section{INTRODUCTION}

High performance motion control using induction motors means controlling the flux and the current producing the torque separately. As the flux measurement in an induction motor has important drawbacks, the flux is often indirectly controlled via an intermediate variable, which is usually the d-axis Park component of the stator current in a reference frame selected in such a way that the rotor flux along the $\mathrm{q}$ axis is equal to zero. Because of its dependence on the motor model, this flux control method is naturally sensitive to parameter uncertainties. These uncertainties are due to the saturation of the inductances, to the temperature and the skin effect which alter the values of the stator and rotor resistance. The rotor resistance usually plays an important role in the field-oriented control of induction motors, but it is also a parameter which is very difficult to determine precisely, particularly in squirrel-cage induction motors.

Parameter uncertainties imply errors on the flux amplitude and orientation with the following consequences.

- The system can become unstable when the orientation error is too large.

- An additional stator current is necessary to develop a given torque, which increases the system losses.

Classical indirect field-oriented control [3] is highly sensitive to uncertainties in the rotor resistance. This is mainly due to the method of computing the stator electrical frequency from the mechanical speed added to an estimation of the slip frequency. However, the stator frequency can also be directly determined from stator voltage and current [2,3]. This second method is not sensitive to uncertainties on the rotor resistance, but is sensitive to uncertainties on the stator resistance and on inductances. As these two methods each have some advantages and some

DOI : 10.5121/ijaia.2013.4103 
drawbacks, it is interesting to combine the two in order to compute the stator frequency [3]. Fuzzy logic is then used to combine the two methods to obtain a compromise which reduces the flux control sensitivity to electrical parameter errors at each operating point. In this paper, the performance of the proposed control algorithm, in particular, its robustness against parameter uncertainties. It should be noted that, in the proposed control algorithm, fuzzy logic is only used to combine two models and that we consider classical speed and current controllers [proportional integral (PI) or integral proportional (IP)] [1, 2]. This approach is unusual, as many authors use fuzzy logic to develop fuzzy controllers instead of classical controllers $[4,5]$. Fuzzy logic is also used by some authors to estimate a parameter $[6,7]$.

\section{MOTOR MODELING}

In a generalized two-ax reference frame, the electrical equations of an induction machine are:

$$
\mid \begin{aligned}
& \dot{\psi}_{s d}=\omega_{s \cdot} \cdot \psi_{s q}-R_{s} \cdot i_{s d}+u_{s d} \\
& \dot{\psi}_{s q}-\omega_{s \cdot} \cdot \psi_{s d}-R_{s} \cdot i_{s q}+u_{s q} \\
& \dot{\psi}_{r d}-\omega_{s r} \cdot \psi_{r q}-R_{r} \cdot i_{r d} \\
& \dot{\psi}_{r q}=-\omega_{s r} \cdot \psi_{r d}-R_{r} \cdot i_{r q}
\end{aligned}
$$

The electromagnetic torque is given by

$$
T_{e m}=p \cdot \frac{M}{L_{r}}\left(i_{s q} \psi_{r d}-i_{s d} \psi_{r q}\right)
$$

The fluxes are related to the currents by the following equations:

$$
\begin{aligned}
& \psi_{s d}=L_{s \cdot i_{s d}}+M \cdot i_{r d} \\
& \psi_{r d}=M \cdot i_{s d}+L_{r} \cdot i_{r d} \\
& \psi_{s q}-L_{s} \cdot i_{s q}+M \cdot i_{r q} \\
& \psi_{r q}-M \cdot i_{s q}+L_{r} \cdot i_{r q}
\end{aligned}
$$

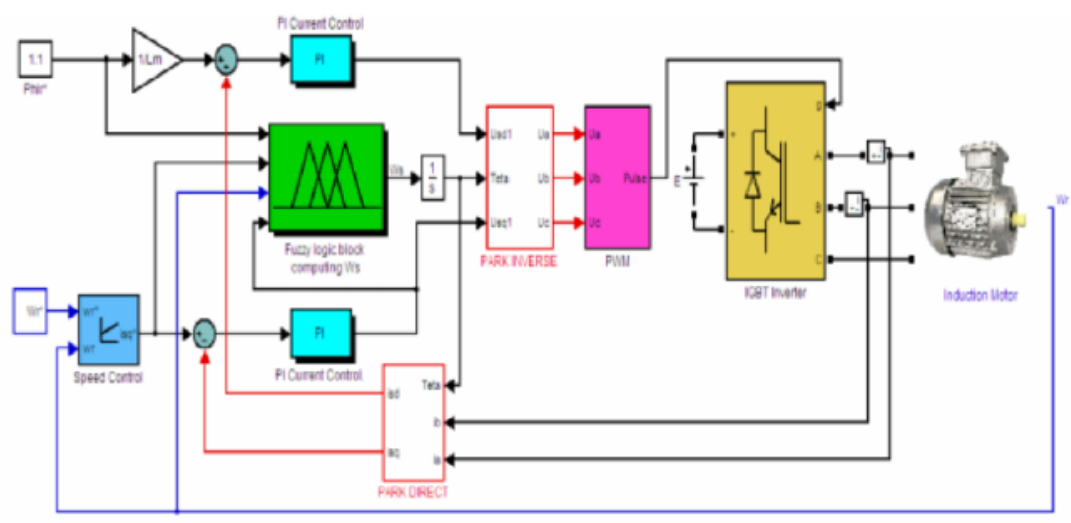

Figure 1.Indirect field-oriented control scheme of an IM 


\section{Control strategy}

The position $\theta$ of the Park reference frame, which ensures the field orientation $\left(\psi_{\mathrm{rq}}=0\right.$ and, $\psi_{\mathrm{rd}}=$ $\left.\psi_{\text {ref }}\right)$ in an indirect control strategy, is computed by integrating the instantaneous stator electrical frequency $\omega_{\mathrm{s}}=p . \Omega_{\mathrm{m}}+\omega_{\mathrm{sr}}$. Generally, the estimation of the slip frequency $\omega_{\mathrm{sr}}$ is obtained by using the rotor $q$ axis (1d) of the motor model, which gives the stator electrical frequency (superscript ${ }^{*}$ indicates an estimated parameter)

$$
\omega_{s 1}=p \cdot \Omega_{m}+\omega_{s r}=p \cdot \Omega_{m}+\frac{M^{*} \cdot R_{r}^{*}}{L_{r}^{*}} \cdot \frac{i_{s q}}{\psi_{r e f}}
$$

The main drawbacks to using (4) are its dependence on the value of the rotor resistance $R_{r}$, which varies with temperature, and its dependence on the value of the magnetizing inductance $M$ which varies with magnetic saturation. The stator electrical frequency can also be determined from the stator q-axis equation of the motor model. By eliminating $i_{r d}$ and $i_{r q}$ in (3) and eliminating $\psi_{\mathrm{sd}}$ and $\psi_{\mathrm{sq}}$ between (3) and (1b), and then by setting $\psi_{\mathrm{rd}}=\psi_{\mathrm{ref}}$, and $i_{s d}=i_{\text {sdref }}=\psi_{\mathrm{ref}} / M^{*}$ in (1b), a direct estimation of the stator electrical frequency is obtained [2], [3] ( $s$ is the Laplace operator)

$$
\omega_{s 2}=\frac{u_{s q}-\left(R_{s}^{*}+\sigma^{*} \cdot L_{s}^{*} \cdot s\right) \cdot i_{s q}}{\frac{L_{s}^{*}}{M^{*} \cdot \psi_{r e f}}}
$$

The advantage of (5) is that it is independent of the rotor resistance $\mathrm{R}_{\mathrm{r}}$. However, (5) depends on the stator resistance, but this parameter is quite easy to determine precisely, and on the derivative of the q-axis current $i_{s q}$. Experiments show that this derivative term can be neglected for the tested motor. The indirect field oriented control scheme considered in this paper is shown in Fig.1. The decoupling terms and the $d, q$ reference frame speed $\omega_{\mathrm{s}}$ are computed with the reference values of the flux and currents. In fact, the reference values give predicted values of the currents in the motor and they are less noisy than the measured values. Moreover, the stability of the system is increased, as shown in [8].

As both methods (4) and (5) for computing $\omega_{\mathrm{s}}$ each have some advantages and drawbacks, it is suggested to compute $\omega_{\mathrm{s}}$ by using a combination of two methods:

$$
\begin{gathered}
\omega_{s}=\left(1-K_{\omega}\right) \cdot \omega_{s 1}+K_{\omega} \cdot \omega_{s 2} \\
0 \leq K_{\omega} \leq 1
\end{gathered}
$$

The value of $K_{\omega}$ is determined by the fuzzy logic blocks shown in the control scheme of figure1.

\section{SENSITIVITY TO PARAMETER UNCERTAINTIES}

\subsection{Flux amplitude and orientation errors}

As the flux is controlled by using models, errors in the electrical parameters imply errors in the flux. These errors can be studied in steady-state conditions. The electrical equations deduced from (1) and (3) are the following: 
International Journal of Artificial Intelligence \& Applications (IJAIA), Vol.4, No.1, January 2013

$$
\begin{aligned}
& u_{s d}=R_{s} \cdot i_{s d}-\sigma \cdot \omega_{s} L_{s} \cdot i_{s q}-\frac{\omega_{s} \cdot M}{L_{r}} \cdot \psi_{r q} \\
& u_{s q}=\sigma \cdot \omega_{s} L_{s} \cdot i_{s d}+R_{s} \cdot i_{s q}+\frac{\omega_{s} \cdot M}{L_{r}} \cdot \psi_{r d} \\
& i_{s d}=\frac{1}{M \cdot \psi_{r d}}-\frac{\omega_{s r} \cdot L_{r}}{M \cdot R_{r}} \cdot \psi_{r q} \\
& i_{s q}=\frac{\omega_{s r} \cdot L_{r}}{M \cdot R_{r}} \cdot \psi_{r d}+\frac{1}{M} \cdot \psi_{r q}
\end{aligned}
$$

From $(7 \mathrm{c})$, and when the $i_{s d}$ current controller includes an integral action, we can write:

$$
i_{s d}=i_{s d r e ́ f}=\frac{1}{M{ }^{*}} \cdot \psi_{r e ́ f}=\frac{1}{M} \cdot \psi_{r d}-\frac{\omega_{s r} \cdot L_{r}}{M \cdot R_{r}} \cdot \psi_{r q}
$$

When $K_{\omega}=0$, it follows from (4) and by taking into account that the $i_{s q}$ current controller includes an integral action:

$$
i_{s q}=i_{s q r e f}=\frac{\omega_{s r} \cdot L_{r}^{*}}{M^{*} \cdot R_{r}^{*}} \cdot \psi_{r e f}=\frac{\omega_{s r} \cdot L_{r}}{M \cdot R_{r}} \cdot \psi_{r d}+\frac{1}{M} \cdot \psi_{r q}
$$

When $K_{\omega}=1$, it follows from (5) and by taking into account that the $i_{s q}$ current controller includes an integral action:

$$
i_{\text {sqref }}=\frac{u_{s q}-\frac{\omega_{s} \cdot L_{s}^{*}}{M^{*} \cdot \psi_{r e f}}}{R_{s}^{*}}=\frac{\omega_{s r} \cdot L_{r}}{M \cdot R_{r}} \cdot \psi_{r d}+\frac{1}{M} \cdot \psi_{r q}
$$

Equations (7) and (3), associated with the control algorithm, yield equations of the following form:

$$
\begin{aligned}
& C . \psi_{\text {ref }}=A_{1} \cdot \psi_{r d}-B_{1} \cdot \psi_{r q} \\
& D . \psi_{\text {ref }}=B_{2} \cdot \psi_{r d}+A_{2} \cdot \psi_{r q}
\end{aligned}
$$

The values of $A_{1}, A_{2}, B_{1}, B_{2}, C$, and $D$ depend on the control strategy. So, (8) and (11a) yield:

$$
\mid \begin{aligned}
& C=\frac{1}{\lambda r^{*}} \\
& B_{1}=\omega_{s T} \cdot \frac{r_{r}}{\lambda r F_{T} \cdot} \\
& 1_{1}-\frac{1}{\lambda r}
\end{aligned}
$$


In (11b), $A 2, B 2$ and $D$ have the following forms:

$$
\left\{\begin{array}{l}
A_{2}=\left(1-K_{\omega}\right) \cdot A_{2}^{\prime}+K_{\omega} \cdot A_{2}^{\prime \prime} \\
B_{2}=\left(1-K_{\omega}\right) \cdot B_{2}^{\prime}+K_{\omega} \cdot B_{2}^{\prime \prime} \\
D=\left(1-K_{\omega}\right) \cdot D^{\prime}+K_{\omega} \cdot D^{\prime \prime}
\end{array}\right.
$$

So, when $\mathrm{K}_{\omega}=0$, (9) and (11 b) yield:

$$
\left\{\begin{array}{l}
D^{\prime}-\frac{\omega_{s r} \cdot I_{r}^{*}}{M I^{*} \cdot R_{r}^{*}} \\
B_{2}^{\prime}=B_{1}=\frac{\omega_{s r} \cdot L_{r}}{M I \cdot R_{r}} \\
A_{2}^{\prime}-A_{1}-\frac{1}{M I}
\end{array}\right.
$$

When $\mathrm{K}_{\omega}=1$, by eliminating $U_{s q}$ in (10) via (7b), and then by eliminating $i_{s d}$ and $i_{s q}$ via (7c) and (7d), you find by identification with (11b)

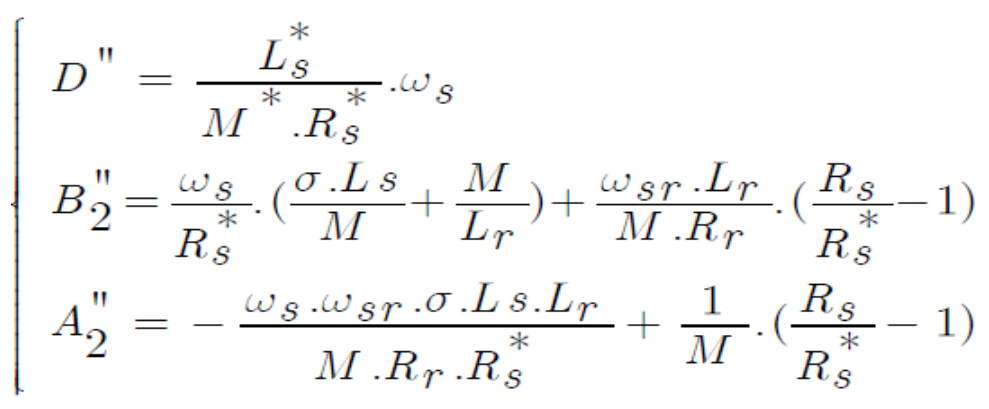

The equations in (11) allow one to determine the errors in the flux, which yield

$$
\left\{\begin{array}{l}
\left(\frac{\psi_{\text {rd }}}{\psi_{\text {ref }}}\right)=\frac{B_{1} \cdot D+A_{2} \cdot C}{A_{1} \cdot A_{2}+B_{1} \cdot B_{2}} \\
\left(\frac{\psi_{r q}}{\psi_{\text {ref }}}\right)=\frac{A_{1} \cdot D-B_{2} \cdot C}{A_{1} \cdot A_{2}+B_{1} \cdot B_{2}}
\end{array}\right.
$$

From (16), the following expressions for the errors, due to parameter uncertainties, in the flux amplitude $\psi \mathrm{r}$ and orientation $\rho$ can be determined: 


$$
\begin{aligned}
& \frac{\psi_{r}}{\psi_{\text {ref }}}=\sqrt{\frac{\left(B_{1} \cdot D+A_{2} \cdot C\right)^{2}+\left(A_{1} \cdot D-B_{2} \cdot C\right)^{2}}{\left(A_{1} \cdot A_{2}+B_{1} \cdot B_{2}\right)^{2}}} \\
& \rho=\arctan \left(\frac{\psi_{r q}}{\psi_{r d}}\right)=\arctan \left(\frac{A_{1} \cdot D-B_{2} \cdot C}{B_{1} \cdot D+A_{2} \cdot C}\right)
\end{aligned}
$$

The electromagnetic torque expression, when there are parameter uncertainties, is deduced from (2) by eliminating the currents via (7c) and (7d), and by taking into account equation (17):

$$
T_{c m}=\frac{p \cdot \omega_{s r}}{R_{r}} \cdot \frac{\left(B_{1} \cdot D+A_{2} \cdot C\right)^{2}+\left(A_{1} \cdot D-B_{2} \cdot C\right)^{2}}{\left(A_{1} \cdot A_{2}+B_{1} \cdot B_{2}\right)^{2}} \cdot \psi_{r e f}^{2}
$$

\subsection{Effects of Saturation}

The previous expressions (7) - (19) are computed from the linear motor model without any saturation effect. As errors in the stator and rotor resistances imply errors in the real value of the flux and, thus, alter the value of the magnetizing inductance, a simple model is introduced into the sensitivity analysis, which is useful in representing the variations of $\mathrm{M}$. This model uses two parameters, a linear one $\beta$ for the air gap and an exponent $\alpha$ for the core saturation $[9,10]$

$$
\begin{aligned}
& i_{m N}=\beta \psi_{s N}+(1-\beta) \cdot \psi_{s N}^{\alpha} \\
& M_{N}=\frac{\psi_{s N}}{i_{m N}}
\end{aligned}
$$

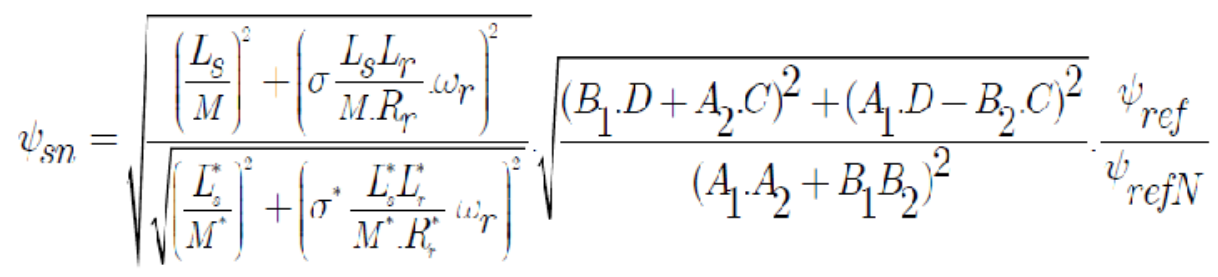

Where $i_{m N}, \quad \psi_{s N}$, and $M_{N}$ are the normalized values of the magnetizing current $i_{m}=\sqrt{\left(i_{s d}+i_{r d}\right)^{2}+\left(i_{s q}+i_{r q}\right)^{2}}$ ), the stator flux $\left(\psi_{s}=\sqrt{\left(\psi_{s d}+\psi_{s q}\right)^{2}}\right.$ ), and $M . \psi_{s N}$ is related to the rotor flux by relation (22), where $\psi_{\text {refN }}$ is the nominal reference value of the rotor flux.

Equation (21) is the static inductance. Since the sensitivity analysis considers only steady-state situations, the dynamic inductance is not taken into account [11]. 
Both parameters $\beta$ and $\alpha$ required by (20) are estimated from terminal voltage and current measurements on the unloaded machine [10]. Fig.2 shows the $1500 \mathrm{~W}$ tested motor and the analytical expression. The analytic expression using the fitted parameter values agrees around the nominal flux value.

To obtain the flux amplitude and orientation errors, for each operating point determined by fixed values of $\omega_{\mathrm{m}}$ and Tem, a system of two equations (19) and (21) with two unknown variables $\omega_{\text {sr }}$ and $M$ has to be solved. As the system is strongly nonlinear, it must be solved numerically. The algorithm is the following.

* An error is introduced on an estimated parameter.

* Values of $\omega_{\mathrm{m}}$ and $T_{e m}$ are fixed.

$* M$ is fixed at an initial value.

* Equation (19) is solved to find $\omega_{\text {sr }}$.

* With this value of $\omega_{\mathrm{sr}},(22),(20)$, and (21) are computed.

* Equation (21) gives a new value of $M$ : if this value is nearly identical to the previous value, then (17) and (18) are computed, if not, we start again at point 4 by considering a new value of $M$ which is obtained by computing an average between the last value of $M$ and its previous values.

This simple algorithm converges very rapidly and gives good results confirmed by simulations results. It may be noticed that, in this study, it is assumed that the mechanical speed is correctly measured using a speed or a position sensor.
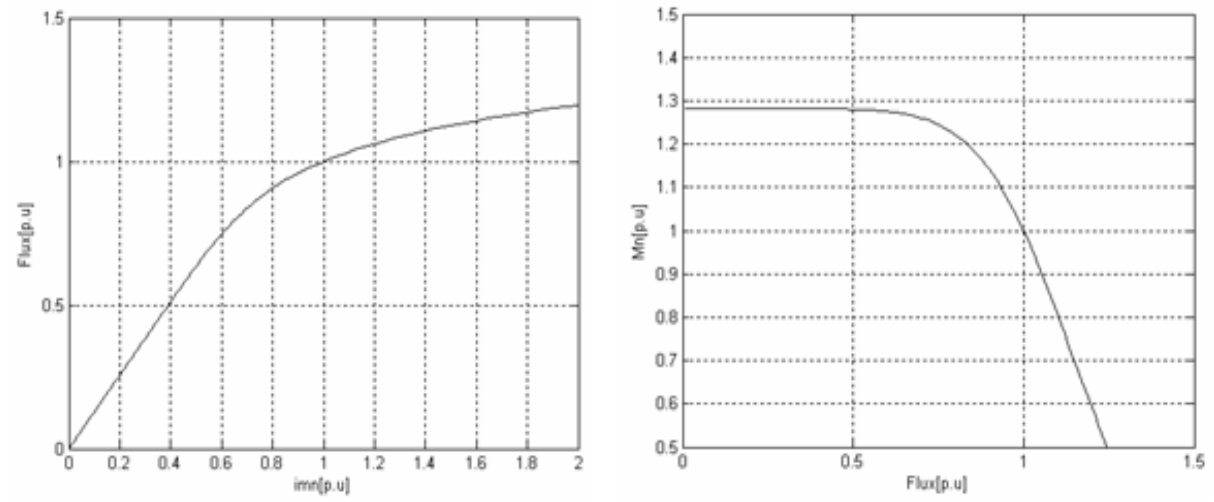

Figure 2. Saturation model Flux versus magnetizing current and magnetizing inductance versus flux $(\alpha=$ 8.8 and $\beta=0.78$ )

- If $K_{\omega}=0: \omega_{\mathrm{s}}$ is computed via the classical method (4), and expressions (16 -19) reduce to the expressions determined in (10 -13).

The theoretical analysis confirms that, in this case, the flux control is highly sensitive to errors in $R_{r}$ and that this sensitivity is independent of the rotor speed.

- If $K_{\omega}=1: \omega_{\mathrm{s}}$ is computed from model (5).

From the theoretical analysis, the following expression for the flux orientation error is obtained: 


$$
\rho=\arctan \left(\frac{\omega_{S}\left(1-\frac{L_{S}}{L_{S}^{*}}\right)-\omega_{S r} \frac{L_{r}}{R_{r} L_{S}^{*}}\left(R_{S}-R_{S}^{*}\right)}{\omega_{S} \omega_{S r} \frac{L_{r}}{R_{r}}\left(1-\sigma \frac{L_{S}}{L_{S}^{*}}\right)+\frac{1}{L_{S}^{*}}\left(R_{S}-R_{S}^{*}\right)}\right)
$$

$$
\text { And if } \omega_{\mathrm{sr}}=0 ; \quad \rho=\arctan \left(p . \Omega_{m} \frac{L_{S}^{*}-L_{S}}{R_{S}^{*}-R_{S}}\right)
$$

Equation (24) shows that, when $\omega_{s r}=0$ and when there is an error in $L_{s}$, the flux orientation error increases with speed and tends toward $\pi / 2$, which will, of course, affect system stability. This result indicates that $K_{\omega}=1$ would be a bad choice.

\subsection{Stator current variation}

The amplitude and orientation errors cannot easily be measured experimentally. But the variations of the stator current allow one to make directly the link between the theory and experiments. These variations appear when there is an error in the flux. The stator current variation is defined by:

$$
\Delta i_{S}=\frac{i_{S}-i_{s i}}{i_{s i}}
$$

Where $i_{s i}$ is the ideal current absorbed when there is no parameter error, and $i_{s}$ (the actual current absorbed). From (7c) and (7d), with $\psi_{r q}=0$ and $\psi_{r d}=\psi_{r e f}$, we get the following expression for the current $i_{s i}$ :

$$
i_{s i}=\sqrt{i_{s d i}^{2}+i_{s q i}^{2}}=\frac{\psi_{r e f}}{M^{*}} \sqrt{1+\left(\frac{\omega_{s r i} \cdot L_{r}^{*}}{R_{r}^{*}}\right)^{2}}
$$

From (2) and (3), with $\psi_{r q}=0$ and $\psi_{r d}=\psi_{r e f}$, the expression of $\omega_{\text {sri }}$ is:

$$
\omega_{s r i}=\frac{T_{e m} \cdot R_{r}^{*}}{p \cdot \psi_{r e f}^{2}}
$$

Equations (2) and (3) yield: 


$$
i_{s}=\sqrt{i_{s d}^{2}+i_{s q}^{2}}=\sqrt{\left(\frac{1}{M}\right)^{2}+\left(\frac{\omega_{s r} \cdot L_{r}}{M \cdot R_{r}}\right)^{2}} \cdot \sqrt{\psi_{r d}^{2}+\psi_{r q}^{2}}
$$

By taking into account expression (9), it becomes:

$$
i_{s}=\frac{\psi_{\text {ref }}}{M^{*}} \sqrt{1+\left(\frac{\omega_{s r i} \cdot L_{r}^{*}}{R_{r}^{*}}\right)^{2}} \cdot \sqrt{\frac{\left(B_{1} \cdot D+A_{1} \cdot C\right)^{2}+\left(A_{1} \cdot D-B_{2} \cdot C\right)^{2}}{\left(A_{1} \cdot A_{2}+B_{1} \cdot B_{2}\right)^{2}}}
$$

With saturation

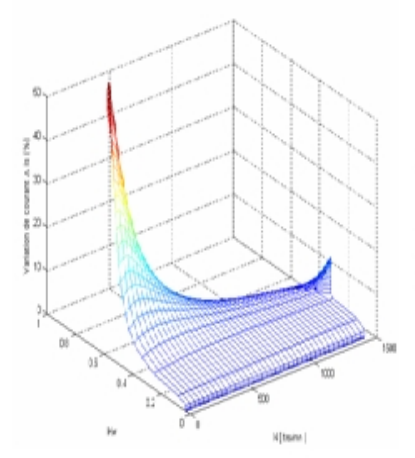

without saturation

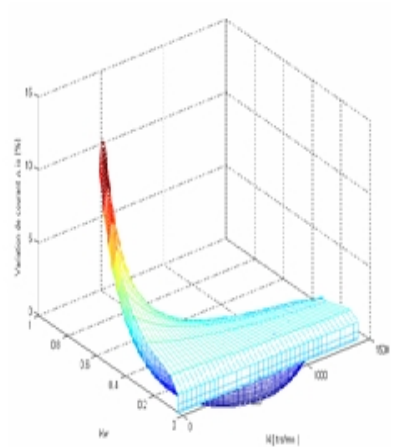

Figure 3. Theoretical results, stator current variation versus $K \omega$ and mechanical speed when $\mathrm{Rr}=2 \mathrm{R}_{\mathrm{r}}$ * and for a 10 N.m torque with and without saturation effect.

Figure 3 shows the stator current variation as a function of $\mathrm{K}_{\omega}$, and of the mechanical speed. The parameters of the tested motor are given in the Appendix. In Fig. 3, an error of $100 \%$ is introduced in the rotor resistance $\left(R_{r}=2 R r^{*}\right)$. The curves of figure 3 are obtained by considering an electromagnetic torque of $10 \mathrm{~N} . \mathrm{m}$ close to the rated value of the tested motor, which corresponds to the worst case as regards the flux control sensitivity to uncertainties on the rotor resistance. In figure 3(a), the curves are obtained by considering the magnetic saturation, whereas the curves in 3(b) are obtained without considering any saturation. These figures show that saturation strongly influences the flux control sensitivity, and that a small value of $\mathrm{K}_{\omega}$, is sufficient to significantly reduce the over current when $R_{r}=2 R r^{*}$. It can also be seen that, in figure 3(a), the current variation is positive. Working at constant flux is, therefore not optimal.

\subsection{Determination of $K_{\omega}$ using Fuzzy logic}

The value of $K_{\omega}$ will naturally be chosen to reduce the sensitivity of the flux control shown in Fig. 1 to the errors in $R r$, but theoretical analysis shows that $K_{\omega}$ must also be chosen to avoid a too high sensitivity to the value of the inductances. $K_{\omega}$ will, thus, be a function of the measured speed $\omega_{\mathrm{m}}$ and of $\omega_{\text {sr }}$. Theoretical analysis shows that $K_{\omega}$ must be very small when the slip frequency $\omega_{\text {sr }}$ is low or when the mechanical speed $\omega_{\mathrm{m}}$ is high. On the other hand, $\mathrm{K}_{\omega}$ must be large when $\omega_{\mathrm{sr}}$ is high and when $\omega_{\mathrm{m}}$ is low (as shown in Fig. 3). Two input variables for the fuzzy logic block, $\omega_{\mathrm{sr}}$ estimated from (4) and $\omega_{\mathrm{m}}$ which is measured must be, therefore, considered. Two fuzzy sets for 
these fuzzy variables, Zero $(Z)$ and $\operatorname{Big}(B)$, are also considered. The determination of $K_{\omega}$ in the fuzzy logic block of Fig. 1 is then achieved as follows.

a) Fuzzification: The chosen membership functions of the normalized variables are given in Fig.4 (a), (b).

$$
X_{r}=\left|\frac{\omega_{s r}}{\omega_{s r} \max }\right|, X_{m}=\left|\frac{\omega_{m}}{\omega_{m} \max }\right|
$$

b) Inference: The chosen fuzzy rules are IF $X_{s r}$ is Band $X_{m}$ is $Z$ THEN $X_{k}$ is $B$, or IF $X_{s r}$ is $Z$ or $X_{m}$ is $B$ THEN $X_{k}$ is $Z$.

When $\omega_{s r} \omega_{m}<0, \mathrm{~K}_{\omega}$ is equal to zero. This means that, during braking operations, (6) is reduced to (4), as considered in this paper, mainly the motor operation.

c) Defuzzification: The membership function of the output variable $X_{k}$, is shown in Fig. 4(c). The fuzzy value $\mathrm{K}_{\omega}$ of the output variable is defuzzified using the "center of gravity" method [14]. The member function of $X_{k}$ is chosen to limit the maximum value of $\mathrm{K}_{\omega}$ so as to reduce the sensitivity to uncertainties in $R$., and to obtain a small value of $X_{k}$ close to zero.

Fig. 5 shows the flux amplitude and orientation errors, the stator current variation and the evolution of $\mathrm{K}_{\omega}$ and of the magnetizing inductance (21) when $R r=2 R_{r}{ }^{*}$ as functions of the rotor speed and of the electromagnetic torque. In Fig. $5, \mathrm{~K}_{\omega}=0$, which corresponds to classical field oriented control. In Fig. $6, \mathrm{~K}_{\omega}$, is determined by fuzzy logic; $\omega$ is then computed from (6). The comparison between these two figures shows that the proposed method to compute $\omega$ significantly reduces the flux error.

\section{SIMULATIONS RESULTSAND DISCUSSION}

Figure 7 shows the results of a step in the speed reference from 0 to $500 \mathrm{rpm}$ followed by a torque step of 10 N.m, with optimized parameters.

Fig. 8 and 9, shows the results, when an error of $100 \%$ is introduced in the estimated value of the rotor resistance. In the result of fig. $8 K_{\omega}=0$. And determined by fuzzy logic in the fig.9, the response of the system shown in Fig. 9 is significantly better than the response shown in Fig. 8 because of the following.

$\checkmark$ The additional stator current absorbed due to bad flux control, when the motor is loaded, is $2.3 \%$ in Fig. 9 instead of 24\% in Fig. 8

$\checkmark$ The speed response is faster in Fig. 9. The results confirm the interest of the proposed model combination. 
International Journal of Artificial Intelligence \& Applications (IJAIA), Vol.4, No.1, January 2013

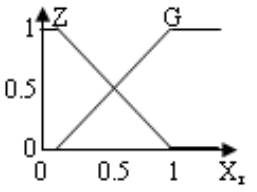

(a)

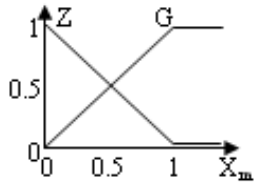

(b)

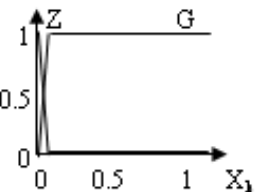

(c)

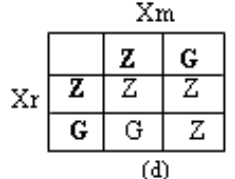

(d)

(e)

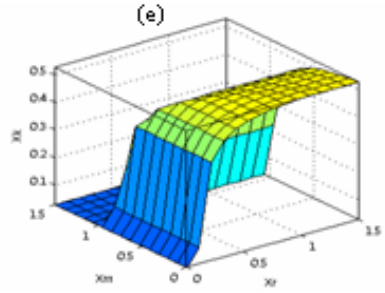

(f).

Figure 4.Membership functions and rule table of the fuzzy controller. (a) Membership functions of input variable $\omega s$ r. (b) Membership functions of input variable $\omega \mathrm{m}$. (c) Membership functions of output variable $(\mathrm{K} \omega)$. (d) Rule table. (e) Fuzzy implication, aggregation and defuzzification method for fuzzy algorithm. (f) Input/output mappings of rules.
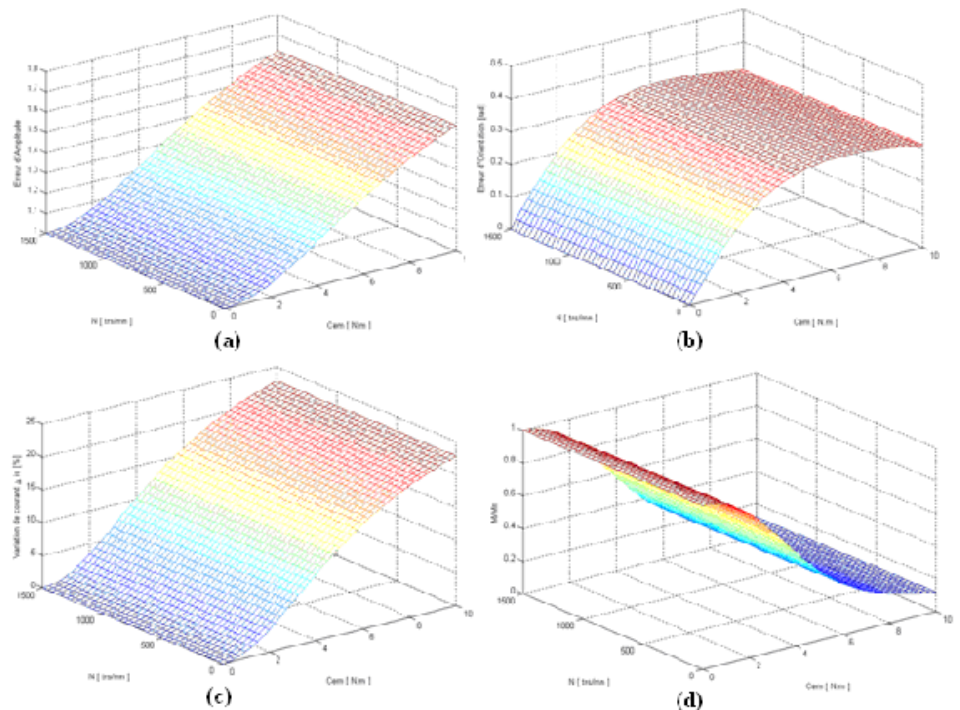

Figure 5.(a): Amplitude flux error, (b) : Orientation flux error (c) current variation and (d) variation of $\mathrm{M}$, when : $\mathrm{Rr}=2 \mathrm{Rr} *$ and $\mathrm{K} \omega=0$. 
International Journal of Artificial Intelligence \& Applications (IJAIA), Vol.4, No.1, January 2013
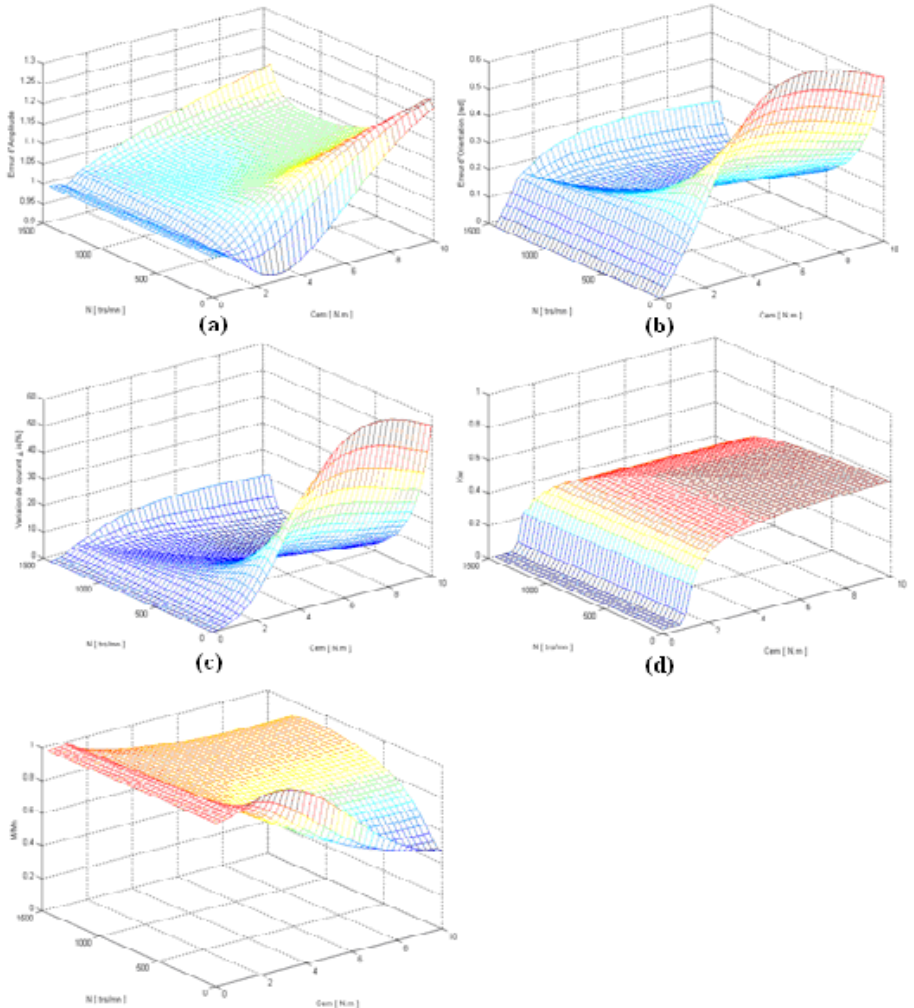

(d)

(e)

Figure 6. (a): Amplitude flux error, (b) : Orientation flux error (c) current variation and (d) variation of $\mathrm{M}$, when : $\mathrm{Rr}=2 \mathrm{Rr} *$ and $\mathrm{K} \omega$ determined by fuzzy logic.

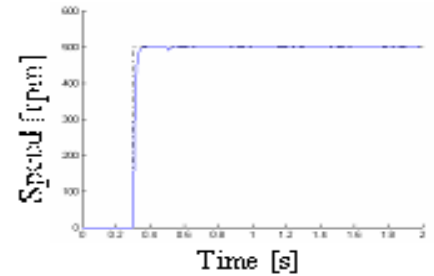

(a)

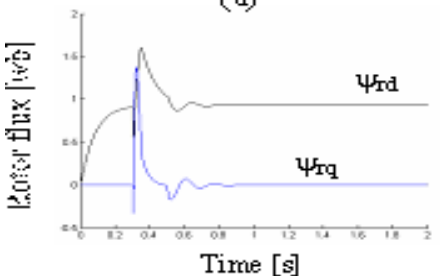

(b)

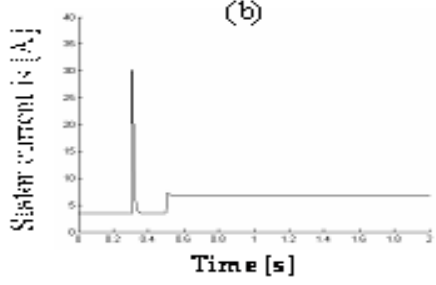

(c)

Figure 7. Simulation results parameters.

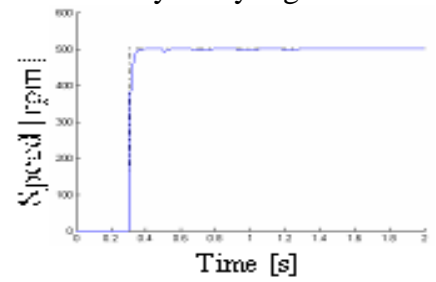

(a)

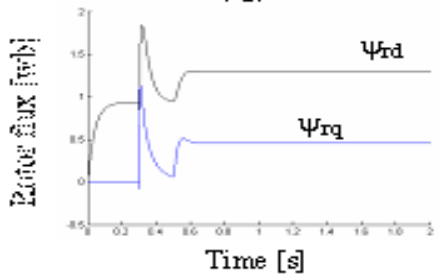

(b)

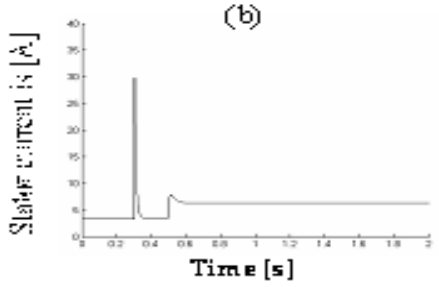

(c)

Figure 8. Simulation results with optimized with error of $100 \%$ on $\mathrm{Rr}$. 
International Journal of Artificial Intelligence \& Applications (IJAIA), Vol.4, No.1, January 2013

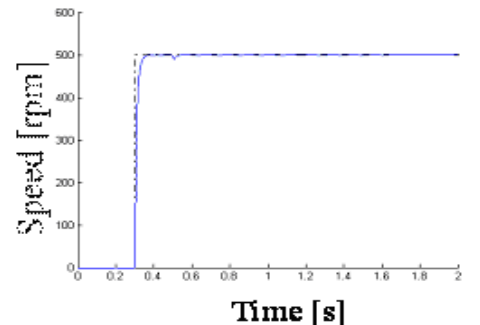

(a)

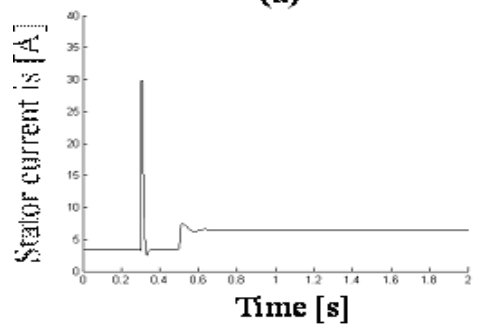

(c)

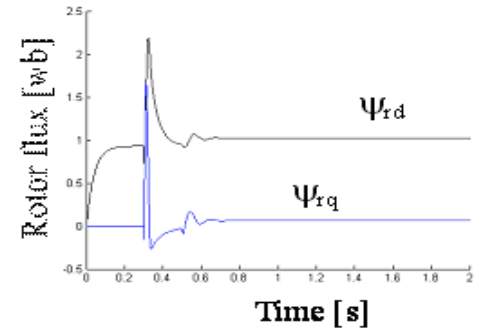

(b)

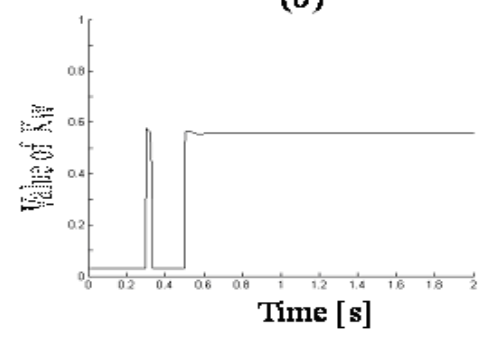

(d)

Figure 9.Simulation results when $\mathrm{Rr}=2 . \mathrm{Rr} *$ and $\mathrm{K} \omega$ determined by fuzzy logic

\section{CONClusion}

In this paper, the performances of an indirect field-oriented control has been studied, the stator electrical frequency being computed by combining two models with the help of fuzzy logic. The results confirm the quality of the proposed method, especially concerning the sensitivity to uncertainties in the rotor resistance. As well as the need to take into account saturation effects in the theoretical analysis and the importance of the variation of the absorbed stator current in characterizing the parameter sensitivity of the control algorithm.

\section{APPENDIX}

\section{Parameters of the induction motor}

\begin{tabular}{ll|ll} 
Rated power & $\mathrm{P}=1500 \mathrm{~W}$ & Stator inductance & $\mathrm{L}_{\mathrm{s}}=0.29 \mathrm{H}$ \\
Rated speed & $\mathrm{n}=1500 \mathrm{rpm}$ & Rotor inductance & $\mathrm{L}_{\mathrm{r}}=0.29 \mathrm{H}$ \\
moment of inertia & $0.0248 \mathrm{Kg} \cdot \mathrm{m}^{2}$ & Mutual inductance & $\mathrm{M}=0.271 \mathrm{H}$ \\
Stator resistance & $\mathrm{R}_{\mathrm{s}}=4.29 \Omega$ & Saturation parameter & $\alpha=8.8$ \\
Rotor resistance & $\mathrm{R}_{\mathrm{r}}=3.6 \Omega$ & & $\beta=0.78$
\end{tabular}

\section{REFERENCES}

[1] S. J. Bassi, M. K. Mishra, and E. E. Omizegba, "automatic tuning of proportional-integral-derivative (pid) controller using particle swarm optimization (pso) algorithm", International Journal of Artificial Intelligence \& Applications (IJAIA), Vol.2, No.4, pp. 25-34, October 2011.

[2] O. Kuljaca, L. Lewis, J. Gadewadikar and K. Horvat, "Dynamic focusing of awareness in fuzzy control systems", International Journal of Artificial Intelligence \& Applications (IJAIA), Vol.2, No.2, pp. 1-19, April 2011.

[3] K. LAROUSSI, M. ZELMAT and M. ROUFF, "Implementation of a Fuzzy Logic System to Tune a PI Controller Applied to an Induction Motor", Advances in Electrical and Computer Engineering, Vol. 9, pp. 107-113, 2009.

[4] Qu Sun, Renhou Li and Ping and Zhang, "Stable and Optimal Adaptive Fuzzy Control of Complex Systems using Fuzzy Dynamic Model", Fuzzy Sets and Systems, No 133, pp. 1 - 17, 2003. 
[5] S. E. Rezgui, and H. Benalla, "high performance controllers for speed and position induction motor drive using new reaching law", International Journal of Instrumentation and Control Systems (IJICS), Vol.1, No.2, pp. 31-46, October 2011.

[6] R. Nirali and S.K. Shah, " Fuzzy Decision Based Soft Multi Agent Controller for Speed Control of Three Phase Induction Motor", International Journal on Soft Computing ( IJSC ), Vol.2, No.3, pp. 46-54, August 2011.

[7] S. Mir, M. E. Elbuluk, and D. S. Zinger, "PI and fuzzy estimators for tuning the stator resistance in Direct Torque Control of induction ma $\neg$ chines," IEEE Trans. Power Electron., Vol. 13, pp. 279-287, Mar. 1998.

[8] B. K. Bose and N. R. Patel, "Quasi fuzzy estimation of stator resistance of induction motor," IEEE Trans. Power Electron., vol. 13, pp. 401-409, May 1998.

[9] B. Robyns, Y. Fu, and F. Labrique, "Stability and robustness study of a fully digital field oriented control system for induction actuators," in Proc. IEEE MELECON'94, Analaya, Turkey, Apr. 1994, pp. 727-730.

[10] F. M. H. Khater, R. D. Lorenz, D. W. Novotny, and K. Tang, "Selection of flux level in field oriented induction machine controllers with consid 7 eration of magnetic saturation effects," IEEE Trans. Ind. Applicat., vol. LA-93, pp. 276-282, Mar./Apr. 1987.

[11] B. Robyns, H. Buyse, and F. Labrique, "Comparison of the sensitivity of the flux control to parameter uncertainties in two induction actuator in direct field oriented control schemes," in Proc. EPE'93, Brighton, U.K, pp. 402-407, Sept. 1993.

[12] B.Chetate and A. Khaldoun, "Commande Indirecte à Flux Rotorique Orienté d'une Machine à Induction avec Adaptation de la Constante de Temps Rotorique par Logique Floue", First International Conference on Electrical Engineering, ICEE'2000.

[13] B. Robyns, F. Labrique, and H. Buyse, "Performance analysis of a simplified field oriented control algorithm for small power induction actuaᄀtors", Math. Comput. Simul, Vol. 38, pp. 389-398, 1995.

[14] A. Hazzab, I.K. Bousserhane, M. Zeebo and P. Pascard, "Real time implantation of fuzzy gain scheduling of PI controller for induction motor machine control", Neural processing letterers pp. 203215 Springer 2006.

\section{Authors}

Mehdi Dhaoui obtained the doctorate thesis in May 2011 in Electrical engineering from ENIG, Tunisia. He works as an associate Professor at the electrical-automatic genius department of the National Engineering School of Gabes, Tunisia. His fields of interest include power electronics, machine drives, automatic control, modeling, identification and optimization. He uses the intelligent technical; Genetic algorithm, Fuzzy logic and Neuronal Network.

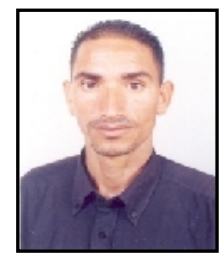

Laakam Makhlouf obtained the master degree of automatic and industrial computer sciences in May 2003 from the National School of Engineering in Sfax ENIS, Tunisia. Currently, he works as a Technologue Teacher, at the Higher institute of Technological Studies of Djerba, Tunisia. His fields of interest include power electronics, machine drives, automatic control and Fuzzy logic.

Lassaâd Sbita obtained the doctorate thesis in July 1997 in Electrical engineering from ESSTT of Tunis, Tunisia. He works as a conferences Professor at the electricalautomatic genius department of the National Engineering School of Gabes, Tunisia. His fields of interest includes power electronics, machine drives, automatic control, modeling, observation and identification.

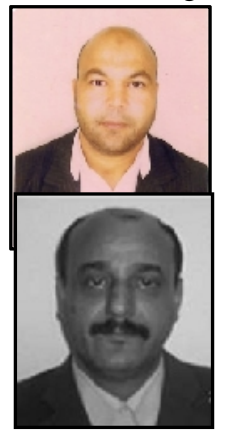

